\title{
DERIVATIONS ON SOME (POSSIBLY NON-SEPARABLE)
}

$$
C^{*} \text {-ALGEBRAS }
$$

\author{
by J. P. SPROSTON
}

(Received 8 July, 1979)

1. Introduction. In an important recent paper [4], G. A. Elliott has given a necessary and sufficient condition for every derivation on a separable $C^{*}$-algebra with identity to be inner. Indeed, Elliott's condition has since been shown, by Akemann and Pedersen, to be equivalent to the $\mathrm{C}^{*}$-algebra being a finite direct sum of $\mathrm{C}^{*}$-algebras which are either homogeneous of finite degree or simple [8, Corollary 3.10].

It is well known $[6,3]$ that every derivation on an $n$-homogeneous $\mathrm{C}^{*}$-algebra with identity is inner, whether or not the algebra is separable. In [6, \$5] examples were given to show that derivations can already be outer in the case of (non-homogeneous) $\mathrm{C}^{*}$-algebras with identity all of whose irreducible *-representations are of dimension 1 or 2 .

In the present paper we investigate derivations on general $C^{*}$-algebras of this latter kind, using an operator field characterization of such algebras based on the work of N. B. Vasil'ev [7]. There are three main results, all to be found in \$3. No assumption of separability is made. (We may perhaps mention that $[6, \S 5$, Example (3)] provides a non-separable $\mathrm{C}^{*}$-algebra with identity, all of whose irreducible *-representations are of dimension 1 or 2 , which has only inner derivations but does not satisfy the condition of Elliott or Akemann-Pedersen.)

This work is a revision of part of the author's Ph.D thesis (University of Newcastle upon Tyne, 1971). The delay in publication is due largely to the desire for more definitive results; despite their continuing absence, the present material is, it is hoped, not without interest. My thanks are due, even after this lapse of time, to Professor J. R. Ringrose for his encouragement and guidance.

2. Operator fields. It is well known [5] that an $n$-homogeneous $C^{*}$-algebra with identity is isometrically $*$-isomorphic to a maximal full algebra of operator fields, the base space being the spectrum of the algebra and the component algebras all being *isomorphic to the $C^{*}$-algebra $M_{n}$ of all complex $n \times n$ matrices.

Throughout this paper $A$ will denote a $C^{*}$-algebra with identity all of whose irreducible *-representations are of dimension 1 or 2 . For such a $\mathrm{C}^{*}$-algebra, a more complicated characterization-as a full but not maximal algebra of operator fields on a slightly different base space-is available from the work of Vasil'ev in [7]. In the following summary we omit detailed proofs where these can be found in, or derived without too much difficulty from, [7].

The spectrum of $A$ is now not in general Hausdorff, and so is unsuitable as a base space. Following [7], let $T^{n}(A)$ denote the space of all equivalence classes of nonzero $n$-dimensional *-representations of $A$, and $P^{n}(A)$ the space of all equivalence classes of nonzero irreducible $n$-dimensional $*$-representations of $A$. It is shown in [7, Theorem 1]

Glasgow Math. J. 22 (1981) 43-56. 
how to topologize $T^{n}(A)$ as a compact Hausdorff space in which $P^{n}(A)$ is an open subset. Then the closure $\overline{P^{n}(A)}$ of $P^{n}(A)$ in $T^{n}(A)$ is itself a compact Hausdorff space. Of course $P^{1}(A)=\overline{P^{1}(A)}=T^{1}(A)$, but in general $P^{2}(A) \varsubsetneqq \overline{P^{2}(A)} \varsubsetneqq T^{2}(A)$.

There is a natural bijection of $T^{2}(A) \backslash P^{2}(A)$ onto the space of unordered pairs of points of $T^{1}(A)$, for each element of $T^{2}(A) \backslash P^{2}(A)$ decomposes as a direct sum of (irreducible) 1-dimensional representations, unique up to order.

Again following [7], we write $X$ for $P^{2}(A)$ (and so $\bar{X}$ for $\overline{P^{2}(A)}$ ) and $Y$ for $P^{1}(A)$; but we prefer to write $\partial X$ for $\bar{X} \backslash X$ (where [7] uses $\dot{X}$ ). Then a point of $\partial X$ may be written as an unordered pair-which we shall write with square brackets, as $\left[y_{1}, y_{2}\right]$-of points $y_{1}, y_{2}$ of $Y$. Again following [7], we write $Z$ for the (open) subset of $\partial X$ consisting of all $\left[y_{1}, y_{2}\right]$ with $y_{1} \neq y_{2}$.

Let $T$ denote the space $\bar{X} \cup Y$, topologized so that a subset $S$ is open in $T$ if and only if $S \cap \bar{X}$ is open in $\bar{X}$ and $S \cap Y$ is open in $Y$. Then $T$ is compact Hausdorff, and we take $T$ as the base space for our operator fields.

For $t$ in $T$, we define the component algebra $A_{t}$ to be the quotient of $A$ by the common kernel of the representations in the class $t$. Thus for $t$ in $X, A_{t}$ is isomorphic to $M_{2}$; and for $t$ in $Y, A_{t}$ is (isomorphic to) $M_{1}=\mathbb{C}$. For $t$ in $\partial X \backslash Z, A_{t}$ is isomorphic to the $C^{*}$-algebra of all scalar multiples of the $2 \times 2$ identity matrix (and hence in fact to $\mathbb{C}$ ); for $t$ in $Z, A_{t}$ is isomorphic to the algebra of all $2 \times 2$ diagonal matrices (which we shall denote by $M_{1} \oplus M_{1}$ ).

Each element $a$ in $A$ yields an operator field on $T$ with values in the $\left\{A_{1}\right\}$, by defining $a(t)$ to be the canonical image of $a$ in $A_{t}$. (We remark, however, that we write $I$ for the identity of $A$ and then $I_{t}$ for the identity $I(t)$ of $\left.A_{t}\right)$. In this way, we identify $A$ with a full algebra of operator fields on $T$. In contrast to the homogeneous case, however, $A$ is not in general a maximal full algebra of operator fields, since, as we now show, it fails to separate the points of $T$.

Firstly, let $t \in \partial X \backslash Z$, so that $t=\left[y_{1}, y_{1}\right]$ for some $y_{1}$ in $Y$. If $b$ is any operator field on $T$ with values in the $\left\{A_{t}\right\}$, then $b(t)=\beta(t) I_{t}, b\left(y_{1}\right)=\beta\left(y_{1}\right) I_{y_{1}}$, for some complex numbers $\beta(t), \beta\left(y_{1}\right)$. (Throughout the paper we shall use a Roman letter to denote an operator field, and the corresponding Greek letter will then be used to indicate the various associated coefficients.) The common kernel of the representations in the class $t$ is the same as that for the class $y_{1}$; so if $b$ is in $A$ then $\beta(t)=\beta\left(y_{1}\right)$.

Secondly, let $t \in Z$, so that $t=\left[y_{1}, y_{2}\right]$ with $y_{1}, y_{2}$ distinct elements of $Y$. Then by [7, p. 149] there exist self-adjoint elements $e_{11}, e_{22}$ (depending on $t$ ) in $A$ such that $e_{11}\left(y_{1}\right)=e_{22}\left(y_{2}\right)=1, e_{11}\left(y_{2}\right)=e_{22}\left(y_{1}\right)=0 ; e_{11}(t)$ and $e_{22}(t)$ are nonzero orthogonal projections in $A_{t}$, and the general element of $A_{t}$ can be written as a complex linear combination of them. In the terminology of [7], $e_{11}(t)$ and $e_{22}(t)$ form a 'basis for $M_{1}+M_{1}$ ' in $A_{t}$; it will be convenient for us to call them matrix units for $M_{1} \oplus M_{1}$ in $A_{t}$. These matrix units are unique up to order, but with $e_{11}, e_{22}$ defined so that $e_{11}\left(y_{1}\right)=e_{22}\left(y_{2}\right)=1, e_{11}\left(y_{2}\right)=e_{22}\left(y_{1}\right)=$ 0 , we may refer unambiguously to $e_{11}(t)$ (resp. $e_{22}(t)$ ) as the matrix unit corresponding to $y_{1}$ (resp. $y_{2}$ ) in $A_{t}$. If then $b$ is any operator field on $T$ with values in the $\left\{A_{t}\right\}$, we may write

$$
b(t)=\beta_{11}(t) e_{11}(t)+\beta_{22}(t) e_{22}(t)
$$


for some complex numbers $\beta_{11}(t), \beta_{22}(t)$. We call $\beta_{11}(t)$ (resp. $\beta_{22}(t)$ ) the coefficient of $b$ at $t$ corresponding to $y_{1}$ (resp. $y_{2}$ ), to indicate which we sometimes write $\beta\left(\bar{y}_{1}, y_{2}\right)$ (resp. $\beta\left(y_{1}, \bar{y}_{2}\right)$ ) for $\beta_{11}(t)$ (resp. $\beta_{22}(t)$ ). If $b$ is in $A$, with $b\left(y_{1}\right)=\beta\left(y_{1}\right) I_{y_{1}}$ and $b\left(y_{2}\right)=\beta\left(y_{2}\right) I_{y_{2}}$, then

$$
b(t)=\beta\left(y_{1}\right) e_{11}(t)+\beta\left(y_{2}\right) e_{22}(t)
$$

in other words, $\beta\left(\bar{y}_{1}, y_{2}\right)=\beta\left(y_{1}\right)$ and $\beta\left(y_{1}, \bar{y}_{2}\right)=\beta\left(y_{2}\right)$.

Recall that if $s, t \in T$ then $A$ separates $s$ and $t$ if, given $u$ in $A_{s}$ and $v$ in $A_{t}$, there exists $a$ in $A$ such that $a(s)=u, a(t)=v$. The above discussion shows that if $b \in A$ then

(i) if $s=\left[s_{1}, s_{2}\right]$ and $t=\left[t_{1}, t_{2}\right]$ both belong to $Z$, with $s_{1}, s_{2}, t_{1}, t_{2}$ distinct elements of $Y$ except that $s_{1}=t_{1}$, then $\beta\left(\bar{s}_{1}, s_{2}\right)=\beta\left(\bar{t}_{1}, t_{2}\right)$;

(ii) if $s=\left[s_{1}, s_{2}\right] \in Z, t=\left[t_{1}, t_{1}\right] \in \partial X \backslash Z$, with $s_{1}=t_{1}$, then $\beta\left(\bar{s}_{1}, s_{2}\right)=\beta\left(t_{1}\right)$;

(iii) if $s=\left[s_{1}, s_{2}\right] \in Z, t=s_{1} \in Y$, then $\beta\left(\bar{s}_{1}, s_{2}\right)=\beta(t)$;

(iv) if $s=\left[s_{1}, s_{1}\right] \in \partial X \backslash Z, t=s_{1} \in Y$, then $\beta(s)=\beta(t)$.

It is then clear that if $s, t$ fall into any of these four categories (or equivalent categories obtained by permuting suffices), then $A$ fails to separate $s$ and $t$. If, on the other hand, $s, t$ fall into none of these categories, then any representation in the class $s$ is disjoint [2, 5.2.1 (ii)] from any representation in the class $t$, and it follows from $[2,4.2 .5]$ that $A$ separates $s$ and $t$.

Lemma 1. With the above notation, let $b$ be an operator field on $T$ with values in the $\left\{A_{t}\right\}$. Then $b$ belongs to $A$ if and only if

(a) $b$ is continuous with respect to $A$ at every point of $T$;

(b) b satisfies the 'separation conditions' (i)-(iv) given above.

Proof. Let $C$ be the collection of all operator fields on $T$ satisfying (a) and (b).

If $b \in A$, (a) holds automatically and (b) follows from the above discussion. So $A \subseteq C$ (and $C$ is itself a full algebra of operator fields on $T$ ). Defining $A(s ; t)=$ $\{(a(s), a(t)): a \in A\}$ and $C(s ; t)=\{(c(s), c(t)): c \in C\}$ we have $A(s ; t) \subseteq C(s ; t)$ for all $s, t \in T$.

If $s, t$ belong to any of the four categories of the separation conditions, it is easy to see that $C(s ; t) \subseteq A(s ; t)$. If $s, t$ belong to none of these categories, then $A$ separates $s$ and $t$, so that $A(s ; t)$ is already all of $A_{s} \times A_{t}$. It follows that $A(s ; t)=C(s ; t)$ for all $s, t \in T$, and so, by [7, VF3, Theorem, p.142], $A=C$. This proves the lemma.

We conclude this section by discussing coordinate neighbourhoods in $T$, and the associated local conditions for continuity with respect to $A$ of an operator field.

Points of $X$. It follows from [5, Theorem 3.1] (cf. [7, VF7, p. 144]) that, just as in the homogeneous case, we can cover $X$ by open coordinate neighbourhoods $\left\{U^{\circ}\right\}$ with each of which is associated a set $\left\{e_{i j}^{\rho}\right\}(i, j=1,2)$ of elements of $A$ such that, for each $t$ in $U^{\rho}$, $\left\{e_{i j}^{o}(t)\right\}$ forms a system of matrix units for $M_{2}$ in $A_{t}$. If $b$ is any operator field on $T$, then for 
$t$ in $U^{\rho}$ we may write

$$
b(t)=\sum_{i, j=1,2} \beta_{i j}^{o}(t) e_{i j}^{o}(t),
$$

where the $\beta_{i j}^{p}(t)$ are complex numbers determined by $b$.

If $t_{0} \in U^{\rho}$, then $b$ is continuous with respect to $A$ at $t_{0}$ if and only if the map

$$
t \mapsto\left\|b(t)-\sum_{i, j=1,2} \beta_{i j}^{p}\left(t_{0}\right) e_{i j}^{p}(t)\right\| \text {. }
$$

is continuous at $t_{0}$. (To see this, note [5, Lemma 1.1] and the fact that $\sum_{i, j=1,2} \beta_{i j}^{p}\left(t_{0}\right) e_{i j}^{p} \in A$.) This condition is in turn equivalent to saying that the four complex-valued maps $t \mapsto \beta_{i j}^{p}(t)$ $(i, j=1,2)$ are all continuous at $t_{0}$. We can also derive this as in [6, p. 524] (where the maximality of $A$ was, incidentally, unnecessarily adduced in proving sufficiency).

Points of $Y$. Points of $Y$ give rise only to a trivial system of matrix units (the identity) which extends trivially in any neighbourhood. If $t_{0} \in Y$ and $b$ is any operator field on $T$, so that $b(t)=\beta(t) I_{t}$ for any $t \in Y$ with $\beta(t)$ a complex number, then $b$ is continuous with respect to $A$ at $t_{0}$ if and only if the map $t \mapsto\left\|b(t)-\beta\left(t_{0}\right) I_{t}\right\|$ is continuous at $t_{0}$, which in turn holds if and only if the map $t \mapsto \beta(t)$ is continuous at $t_{0}$.

Points of $Z$. If $t=\left[y_{1}, y_{2}\right] \in Z$, it follows from our earlier discussion and [7, VF7, p. 144] that there is a neighbourhood $O_{t}$ (in $\bar{X}$ ) of $t$ and self-adjoint elements $e_{11}, e_{22}$ (depending on $t$ ) in $A$ such that $e_{11}(s)$ and $e_{22}(s)$ are matrix units for $M_{1} \oplus M_{1}$ in $A_{s}$ for all $s \in O_{t}$ (see [7, p. 149]). (In particular, $O_{t} \cap(\partial X \backslash Z)=\varnothing$, so that $Z$ is open in $\partial X$ ). If $s=\left[u_{1}, u_{2}\right]$ is any point in $O_{t} \cap Z$, then $u_{1}, u_{2}$ are distinct elements of $Y$, but it is important to remember that the matrix units $e_{11}(s)$ and $e_{22}(s)$ may correspond (in the sense defined earlier) possibly to $u_{1}, u_{2}$ respectively, possibly to $u_{2}, u_{1}$ respectively.

Further, for each $x$ in $O_{t} \cap X$ there is a neighbourhood $V_{t, x}$ of $x$ (contained in $\left.O_{t} \cap X\right)$ and elements $e_{12}, e_{21}$ (depending on $t$ and $\left.x\right)$ in $A$ such that $\left\{e_{i j}(s)\right\}(i, j=1,2)$ form a system of matrix units for $M_{2}$ in $A_{s}$ for all $s \in V_{t, x}$ (for we can find two elements of $A_{x}$ which, together with $e_{11}(x), e_{22}(x)$, form a system of matrix units for $M_{2}$ in $A_{x}$, and then apply [5, Lemma 3.2] or [7, VF6, p. 144]).

To avoid specifying $t$ and $x$, we follow [7] in indexing the $O_{t}$ and $V_{t, x}$ as $O^{\mu}$ and $V^{\mu \gamma}$, and the corresponding elements of $A$ inducing matrix units as $e_{11}^{\mu}, e_{22}^{\mu}, e_{12}^{\mu \gamma}, e_{21}^{\mu \gamma}$ (or collectively as $\left\{e_{i j}^{\mu \gamma}\right\}$, it being then understood that $e_{11}^{\mu \gamma}=e_{11}^{\mu}$ and $e_{22}^{\mu \gamma}=e_{22}^{\mu}$ are both independent of $\gamma$ ).

If $b$ is any operator field on $T$, then for $t$ in $O^{\mu} \cap Z$ we may write

$$
b(t)=\beta_{11}^{\mu}(t) e_{11}^{\mu}(t)+\beta_{22}^{\mu}(t) e_{22}^{\mu}(t),
$$

where $\beta_{11}^{\mu}(t), \beta_{22}^{\mu}(t)$ are complex numbers determined by $b$. If $t=\left[y_{1}, y_{2}\right]$ then $\beta_{11}^{\mu}(t)$, $\beta_{22}^{\mu}(t)$ are equal to $\beta\left(\bar{y}_{1}, y_{2}\right), \beta\left(y_{1}, \bar{y}_{2}\right)$ (in the notation introduced above) but not necessarily in that order. It is also important to note that if $t \in O^{\mu} \cap O^{\nu} \cap Z$, then either $e_{11}^{\mu}(t)=e_{11}^{\nu}(t)$ and $e_{22}^{\mu}(t)=e_{22}^{\nu}(t)$ or $e_{11}^{\mu}(t)=e_{22}^{\nu}(t)$ and $e_{22}^{\mu}(t)=e_{11}^{\nu}(t)$, but that it cannot be assumed that one of these possibilities holds exclusively throughout $O^{\mu} \cap O^{\nu} \cap Z$ (although one will hold exclusively throughout any connected component of $O^{\mu} \cap O^{\nu} \cap Z$ ). 
For $t$ in $O^{\mu} \cap X, t \in V^{\mu \gamma}$ for some $\gamma$, and we may write

$$
b(t)=\sum_{i, j=1,2} \beta_{i j}^{\mu \gamma}(t) e_{i j}^{\mu \gamma}(t),
$$

where the $\beta_{i j}^{\mu \gamma}(t)$ are complex numbers determined by $b$. In fact $\beta_{11}^{\mu \gamma}(t)$ and $\beta_{22}^{\mu \gamma}(t)$ are independent of $\gamma$, just as $e_{11}^{\mu \gamma}(t)=e_{11}^{\mu}(t)$ and $e_{22}^{\mu \gamma}(t)=e_{22}^{\mu}(t)$ are, and so we may write $\beta_{11}^{\mu}(t), \beta_{22}^{\mu}(t)$ respectively-to see this note that $e_{11}^{\mu}(t) b(t) e_{11}^{\mu}(t)$ and $e_{22}^{\mu}(t) b(t) e_{22}^{\mu}(t)$, which are independent of $\gamma$, are just $\beta_{11}^{\mu \gamma}(t) e_{11}^{\mu}(t)$ and $\beta_{22}^{\mu \gamma}(t) e_{22}^{\mu}(t)$ respectively. Consideration of $e_{11}^{\mu}(t) b(t) e_{22}^{\mu}(t)$ and of $e_{22}^{\mu}(t) b(t) e_{11}^{\mu}(t)$ shows similarly that $\left|\beta_{12}^{\mu \gamma}(t)\right|$ and $\left|\beta_{21}^{\mu \gamma}(t)\right|$ are independent of $\gamma$.

The neighbourhoods $V^{\mu \gamma}$ are of the same type as the $U^{\rho}$ constructed earlier, and as before $b$ is continuous with respect to $A$ at a point $t_{0}$ of $V^{\mu \gamma}$ if and only if the maps $t \mapsto \beta_{i j}^{\mu \gamma}(t)(i, j=1,2)$ are all continuous at $t_{0}$. For a point $t_{0}$ of $O^{\mu} \cap Z, b$ is continuous with respect to $A$ at $t_{0}$ if and only if the map

$$
t \mapsto\left\|b(t)-\left(\beta_{11}^{\mu}\left(t_{0}\right) e_{11}^{\mu}(t)+\beta_{22}^{\mu}\left(t_{0}\right) e_{22}^{\mu}(t)\right)\right\|
$$

is continuous at $t_{0}$, which in turn holds if and only if the following two conditions are satisfied:

(ia) the complex-valued maps $t \mapsto \beta_{11}^{\mu}(t), t \mapsto \beta_{22}^{\mu}(t)$ (defined on all of $O^{\mu}$ ) are continuous at $t_{0}$;

(iia) the real numbers $\left|\beta_{12}^{\mu \gamma}(t)\right|,\left|\beta_{21}^{\mu \gamma}(t)\right|$ (defined on $O^{\mu} \cap X$ and independent of $\gamma$ as noted above) $\rightarrow 0$ as $t \rightarrow t_{0}$.

Points of $\partial X \backslash Z$. Points of $\partial X \backslash Z$, like those of $Y$, give rise only to a trivial system of matrix units (the identity) which extends trivially in any neighbourhood. If $t_{0} \in \partial X \backslash Z$ and $N$ is a neighbourhood (in $\bar{X}$ ) of $t_{0}$, then $N$ will in general contain points of $X$, points of $Z$, and other points of $\partial X \backslash Z$. There is no single form of matrix units for all the component algebras $A_{t}(t \in N)$ : for example $N \cap X$ will in general be contained in no single coordinate neighbourhood of any of the types so far constructed. If $t_{0} \in \partial X \backslash Z$ and $b$ is any operator field on $T$, then $b\left(t_{0}\right)=\beta\left(t_{0}\right) I_{t_{0}}$ for some complex number $\beta\left(t_{0}\right)$, and $b$ is continuous with respect to $A$ at $t_{0}$ if and only if the map $t \mapsto\left\|b(t)-\beta\left(t_{0}\right) I_{t}\right\|$ is continuous at $t_{0}$. This condition is in turn equivalent to saying that, given $\varepsilon>0$, there exists a neighbourhood $N_{\varepsilon}$ of $t_{0}$ in $\bar{X}$ such that the following three conditions are satisfied:

(ib) if $t \in N_{e} \cap(\partial X \backslash Z)$, then $\left|\beta(t)-\beta\left(t_{0}\right)\right|<\varepsilon$;

(iib) if $t \in N_{\varepsilon} \cap Z$, then $\left|\beta_{11}(t)-\beta\left(t_{0}\right)\right|$ and $\left|\beta_{22}(t)-\beta\left(t_{0}\right)\right|$ are both less than $\varepsilon$, where $\beta_{11}(t), \beta_{22}(t)$ are the coefficients for $b(t)$ in any matrix-unit system for $A_{t}$;

(iiib) if $t \in N_{\varepsilon} \cap X$, then $\left|\beta_{11}(t)-\beta\left(t_{0}\right)\right|,\left|\beta_{22}(t)-\beta\left(t_{0}\right)\right|,\left|\beta_{12}(t)\right|,\left|\beta_{21}(t)\right|$ are all less than $\varepsilon$, where the $\beta_{i j}(t)(i, j=1,2)$ are the coefficients for $b(t)$ in any matrix-unit system for $A_{t}$.

3. Derivations. Throughout this section, $A$ will denote a $C^{*}$-algebra with identity, all of whose irreducible $*$-representations are of dimension 1 or 2 , identified as in $\$ 2$ with a full algebra of operator fields on $T=\bar{X} \cup Y$; we adopt all the associated notation from $\$ 2$. 
Let $D$ be a derivation on $A$. For each $t$ in $T, A_{t}$ is the quotient $A / J_{t}$ of $A$ by the closed two-sided ideal $J_{t}=\{x \in A: x(t)=0\}$. Since $D\left(J_{t}\right) \subseteq J_{t}[2,1.9 .11(\mathrm{~d})], D$ induces a well-defined map $D_{t}: A_{t} \rightarrow A_{t}$ given by

$$
D_{t}(a(t))=(D a)(t) \quad(a \in A) .
$$

It is easy to check that $D_{t}$ is a derivation on $A_{t}$. Moreover, $\left\|D_{t}\right\| \leq\|D\|$ : to see this, note that for $a \in A$ we have

$$
\left\|D_{t}\left(a+J_{t}\right)\right\|=\left\|D a+J_{t}\right\| \leq\|D a+b\|
$$

for all $b \in J_{t}$, by definition of the quotient norm. Again since $D\left(J_{t}\right) \subseteq J_{t}$, it follows that

$$
\left\|D_{t}\left(a+J_{t}\right)\right\| \leq\|D a+D b\| \leq\|D\|\|a+b\|
$$

for all $b \in J_{t}$, so that

$$
\left\|D_{t}\left(a+J_{t}\right)\right\| \leq\|D\| \inf \left\{\|a+b\|: b \in J_{t}\right\}=\|D\|\left\|a+J_{t}\right\| .
$$

It follows that $\left\|D_{t}\right\| \leq\|D\|$ as asserted.

Since, for any $t$ in $T, A_{t}$ is isomorphic to either $M_{1}, M_{2}$, or $M_{1} \oplus M_{1}$, each $D_{t}$ is inner, and so there exists an operator field $b$ on $T$ such that $b(t)$ implements $D_{t}$ for all $t$ in $T$; we may assume, by [1, Corollaire, p. 312], that $\|b(t)\| \leq\left\|D_{t}\right\| \leq\|D\|$ as above. Hence

$$
(D a)(t)=D_{t}(a(t))=b(t) a(t)-a(t) b(t)=(b a-a b)(t) \quad(a \in A, t \in T),
$$

so that $b$ is a bounded operator field $(\|b(t)\| \leq\|D\|$, for all $t)$ implementing $D$. $D$ will be inner if, and only if, for each $t$ in $T$, an element of the centre of $A_{t}$ can be found which, when subtracted from $b(t)$, modifies $b$ into an operator field satisfying the continuity and separation conditions of Lemma $1(\$ 2)$. We investigate continuity first, and separation later.

The centre of $A_{t}$ consists of all scalar multiples of the identity $I_{t}$ in $A_{t}$, except for $t$ in $Z$, where the centre is all of $A_{t}\left(\cong M_{1} \oplus M_{1}\right)$. For $t$ in $Y$, we can subtract $b(t)$ itself, making the operator field zero on $Y$ and so continuous with respect to $A$ on $Y$. The new operator field still implements $D$, for $D_{t}=0$ (because $A_{t}$ is commutative) for all $t$ in $Y$. We can view this as an application to $Y$ of the arguments of [6, Theorem 1] with $n=1$; and the same arguments with $n=2$ applied to coordinate neighbourhoods of the form $U^{\rho}$ and $V^{\mu \gamma}$ deal with $X$ : if, for $t$ in $X$, we subtract from $b(t)$ the trace of $b(t)$, that is,

$$
\frac{1}{2}\left(\beta_{11}^{\rho}(t)+\beta_{22}^{\rho}(t)\right) I_{t} \quad\left(\text { for } t \in U^{\rho}\right)
$$

or

$$
\frac{1}{2}\left(\beta_{11}^{\mu \gamma}(t)+\beta_{22}^{\mu \gamma}(t)\right) I_{t} \quad\left(\text { for } t \in V^{\mu \gamma}\right)
$$

we get an operator field, consistently defined on 'overlaps', which still implements $D$ and is now continuous with respect to $A$ on $X$. These modifications do not affect the boundedness of the operator field implementing $D$. Continuity cannot however always be achieved for $b$ at points of $\partial X$. Our first result, which generalizes the situation of $[6, \S 5$, Example (1)], shows that continuity cannot in general be achieved for $b$ at any point of 
$\partial X \backslash Z$ with a countable base of neighbourhoods; it is possible that the same holds at any point whatever of $\partial X \backslash Z$.

THEOREM 1. Suppose that $\partial X \backslash Z$ contains a point with a countable base of neighbourhoods. Then there exists a derivation $D$ on $A$ which cannot be implemented by an operator field continuous with respect to $A$ on $T$ (so that $D$ is outer).

Proof. Let $\left\{N_{j}\right\}(j=1,2, \ldots)$ be a countable base of open neighbourhoods at $t_{0} \in$ $\partial X \backslash Z$. Replacing $N_{j}$ by $N_{1} \cap N_{2} \cap \ldots \cap N_{j}$ if necessary $(j=1,2, \ldots)$, we may assume that $N_{1} \supseteq N_{2} \supseteq \ldots$. .

Pick $x_{1} \in N_{1} \cap X$. Then $N_{1} \cap X$ is an open neighbourhood of $x_{1}$, and since $\bar{X}$ is compact Hausdorff there is an open neighbourhood $B_{1}$ of $x_{1}$ with $\bar{B}_{1} \subseteq N_{1} \cap X$. Then $t_{0} \notin \bar{B}_{1}$, and there exists an open set $C_{1}$ containing $t_{0}$ with $C_{1} \cap \bar{B}_{1}=\varnothing$. For some $n_{2}(>1)$, $N_{n_{2}} \subseteq C_{1}$; pick $x_{2} \in N_{n_{2}} \cap X$. Again there is an open neighbourhood $B_{2}$ of $x_{2}$ with $\bar{B}_{2} \subseteq N_{n_{2}} \cap X$. Then $\bar{B}_{2} \subseteq C_{1}$, so that $\bar{B}_{2}$ is disjoint from $\bar{B}_{1}$.

Suppose integers $n_{1}, n_{2}, \ldots, n_{m}$ (with $1=n_{1}<n_{2}<\ldots<n_{m}$ ), points $x_{1}, x_{2}, \ldots, x_{m}$, and open sets $B_{1}, B_{2}, \ldots, B_{m}$, have been found such that

$$
x_{j} \in B_{j} \subseteq \bar{B}_{i} \subseteq N_{n_{i}} \cap X \quad(j=1,2, \ldots, m),
$$

with $\bar{B}_{1}, \bar{B}_{2}, \ldots, \bar{B}_{m}$ mutually disjoint. Then $t_{0} \notin \bigcup_{j=1}^{m} \bar{B}_{j}$, and there exists an open set $C_{m+1}$ containing $t_{0}$ with $C_{m+1} \cap\left(\bigcup_{j=1}^{m} \bar{B}_{j}\right)=\varnothing$. For some $n_{m+1}\left(>n_{m}\right), N_{n_{m+1}} \subseteq C_{m+1}$; pick $x_{m+1} \in N_{n_{m+1}} \cap X$. As before there is an open neighbourhood $B_{m+1}$ of $x_{m+1}$ with $\bar{B}_{m+1} \subseteq N_{n_{m+1}} \cap X$. Then $\bar{B}_{m+1} \subseteq C_{m+1}$, and so $\bar{B}_{1}, \ldots, \bar{B}_{m+1}$ are mutually disjoint.

It follows by induction that there exists a sequence $\left\{x_{r}\right\}(r=1,2, \ldots)$ of points of $X$ and open sets $B_{r}$ such that, for some $n_{r}$ with $1=n_{1}<n_{2}<\ldots$,

$$
x_{r} \in B_{r} \subseteq \bar{B}_{r} \subseteq N_{n_{r}} \cap X \quad(r=1,2, \ldots),
$$

and $\bar{B}_{1}, \bar{B}_{2}, \ldots$ are mutually disjoint. Clearly $x_{r} \rightarrow t_{0}$ as $r \rightarrow \infty$ : any neighbourhood of $t_{0}$ contains $N_{n_{r}}$, and hence $x_{r}$, for all sufficiently large $r$.

Each $x_{r}$ lies in some coordinate neighbourhood $U^{o_{r}}$; shrinking each $B_{r}$ if necessary, we may assume that $\bar{B}_{r} \subseteq U^{\rho_{r}}(r=1,2, \ldots)$. For each $r$ there is a continuous function $f_{r}$ defined on $\bar{X}$, taking values in the interval $[0,1]$, such that $f_{r}\left(x_{r}\right)=1$ and $f_{r}(t)=0$ for $t \in \bar{X} \backslash B_{r}$. Define an operator field $b$ on $T$ by putting

$$
b(t)= \begin{cases}f_{r}(t) e_{11}^{\rho_{r}}(t) & \left(t \in B_{r}, r=1,2, \ldots\right), \\ 0 & \left(t \in T \backslash \bigcup_{r} B_{r}\right) .\end{cases}
$$

This operator field is continuous with respect to $A$ at all points of $T$ except $t_{0}$. (Note that any point $s$ in $T$ other than $t_{0}$ has a neighbourhood intersecting at most one $B_{r}$ : there exist disjoint open sets $P_{1}, P_{2}$ containing $s, t_{0}$ respectively, and, for some $r_{0}, P_{2}$ contains $N_{n_{n}}$, and hence $\bar{B}_{r}$, for all $r \geq r_{0}$. But $s$ belongs to at most one of the disjoint sets $\bar{B}_{1}, \bar{B}_{2}, \ldots, \bar{B}_{\mathrm{r}_{0}-1}$, and so $P_{1}$ can be shrunk to give a neighbourhood of $s$ intersecting at most one $\bar{B}_{r}$; working within such a neighbourhood, it is clear from the continuity of $f_{r}$ on 
$\bar{X}$ and its vanishing outside $B_{r}$ that $b$ is continuous with respect to $A$ at $s$.) At $t_{0}$, however, $b$ fails to be continuous with respect to $A$, since although $x_{r} \rightarrow t_{0}$ as $r \rightarrow \infty$, yet $\left\|b\left(x_{r}\right)\right\|=1 \nrightarrow\left\|b\left(t_{0}\right)\right\|=0$.

We show that $b a-a b$ belongs to $A$ whenever $a$ does. Let $a \in A$, with

$$
a(t)=\sum_{i, j=1,2} \alpha_{i j}^{\rho_{j}}(t) e_{i j}^{\rho_{j}}(t) \quad\left(t \in U^{\rho_{r}}\right)
$$

Then

$$
b(t) a(t)-a(t) b(t)= \begin{cases}f_{r}(t) \alpha_{12}^{\rho_{2}}(t) e_{12}^{\rho_{r}}(t)-f_{r}(t) \alpha_{21}^{\rho_{r}}(t) e_{21}^{\rho_{1}}(t) & \left(t \in B_{r}, r=1,2, \ldots\right) \\ 0 & \left(t \in T \backslash \bigcup_{r} B_{r}\right) .\end{cases}
$$

Clearly the operator field $b a-a b$ satisfies the separation conditions of Lemma 1 , since $b(t) a(t)-a(t) b(t)=0$ for all $t$ in $Y \cup \partial X$; and it is continuous with respect to $A$ at all points of $T$ other than $t_{0}$, since $b$ is. Unlike $b$, it is also continuous with respect to $A$ at $t_{0}$ : of the relevant conditions in $\$ 2$, (ib) and (iib) are trivially satisfied, while to satisfy (iiib) it is enough to find, given $\varepsilon>0$, a neighbourhood $N_{\varepsilon}$ of $t_{0}$ such that $\left|f_{r}(t) \alpha_{12}^{\rho_{1}}(t)\right|$ and $\left|f_{r}(t) \alpha_{21}^{\rho_{r}}(t)\right|$ are less than $\varepsilon$ whenever $t \in N_{\varepsilon} \cap U^{\rho_{r}}(r=1,2, \ldots)$. Since $\left|f_{r}(t)\right| \leq 1$ for all $t \in \bar{X}$, we need only have $\left|\alpha_{12}^{\rho_{r}}(t)\right|$ and $\left|\alpha_{21}^{\rho_{1}}(t)\right|$ less than $\varepsilon$ for $t \in N_{\varepsilon} \cap U^{\rho_{r}}$, and now the existence of $N_{\varepsilon}$ is assured by applying the same condition (iiib) to the element $a$ of $A$.

Thus $b$ implements a derivation $D$ on $A$. Suppose that $D$ can be implemented by an operator field $c$ which is continuous with respect to $A$ on $T$. Then $b(t)-c(t)$ belongs to the centre of $A_{t}$ for all $t$ in $T$; in particular, $b\left(x_{r}\right)-c\left(x_{r}\right)=\theta_{r} I_{x_{1}}$ for some complex number $\theta_{r}(r=1,2, \ldots)$, so that $c\left(x_{r}\right)=\left(1-\theta_{r}\right) e_{11}^{\rho_{1}}\left(x_{r}\right)-\theta_{r} e_{22}^{\rho_{2}}\left(x_{r}\right)(r=1,2, \ldots)$. But $c\left(t_{0}\right)=\gamma\left(t_{0}\right) I_{t_{0}}$ for some complex number $\gamma\left(t_{0}\right)$. So, since $x_{r} \rightarrow t_{0}$ as $r \rightarrow \infty$ and $c$ is assumed continuous with respect to $A$ at $t_{0}$, condition (iiib) applied to $c$ gives that both $1-\theta_{r}$ and $-\theta_{r}$ converge to the same complex number $\gamma\left(t_{0}\right)$ as $r \rightarrow \infty$; and this is impossible. This completes the proof of the theorem.

Our next result will be that, with $\partial X \backslash Z$ assumed empty, the desired continuity for $b$ on $Z$ can be achieved if and only if $\bar{X}$ is the Stone-Čech compactification of $X$; this should be compared with [6, §5, Examples (2) and (3)]. (For the record, there should be a minus sign on the lower left entry in the last matrix appearing on p. 534 of [6], but the argument there is not affected). Recall that the Stone-Čech compactification $V$ of a completely regular Hausdorff space $U$ is characterized by the facts that $U$ is dense in $V, V$ is compact, and any bounded continuous complex-valued function on $U$ can be extended to a continuous complex-valued function on $V$.

LEMMA 2. Let $U$ be a completely regular Hausdorff space.

(i) Suppose $V$ is the Stone-Čech compactification of $U$. Let $t_{0} \in V \backslash U$ and let $W$ be any open neighbourhood of $t_{0}$ in $V$. Let $g$ be a bounded continuous complex-valued function defined on $W \cap U$. Then $g$ can be extended to a bounded continuous function on all of $W$.

(ii) Suppose $U$ is dense in a compact Hausdorff space $V$ which is not the Stone-Čech compactification of $U$. Then there exists a bounded continuous complex-valued function $g$ 
defined on $U$ and a point $t_{0}$ in $V \backslash U$ such that $g$ cannot be extended to a bounded continuous function on any neighbourhood of $t_{0}$ in $V$.

Proof. (i) Let $t$ be any point of $W \backslash U$. Then $W$ is an open neighbourhood of $t$ in $V$. Since $V$ is compact Hausdorff, there exists an open neighbourhood $G$ of $t$ in $V$ with $\bar{G} \subseteq W$. Then $\bar{G}$ and $V \backslash W$ are disjoint closed subsets of $V$, and there exists a real-valued continuous function $f$ on $V$, taking the value 1 on $\bar{G}$ and 0 on $V \backslash W$. Define a function $h$ on $U$ by putting

$$
h(x)= \begin{cases}f(x) g(x) & (x \in W \cap U) \\ 0 & (x \in U \backslash W) .\end{cases}
$$

Since $f$ and $g$ are bounded and continuous and $f$ vanishes outside $W, h$ itself is bounded and continuous on $U$. By the Stone-Čech property, $h$ extends to a continuous function $\bar{h}$ on $V$. Since $h$ and $g$ agree on $\bar{G} \cap U, \bar{h}$ yields a continuous extension of $g$ to $(W \cap U) \cup G$ (equal to $g$ on $W \cap U$ and to $\bar{h}$ on $G$ ).

If we carry out this construction for each $t$ in $W \backslash U$, the functions $\bar{h}$ agree on 'overlaps'; for suppose $t^{\prime} \in W \backslash U$, and form the corresponding $G^{\prime}, h^{\prime}, \bar{h}^{\prime}$ as before. Then if $t^{\prime \prime} \in\left(G \cap G^{\prime}\right) \backslash U, t^{\prime \prime}$ is the limit of a net $\left(x_{\sigma}\right)$ of points of $\left(G \cap G^{\prime}\right) \cap U$, and since $\bar{h}\left(x_{\sigma}\right)=\bar{h}^{\prime}\left(x_{\sigma}\right)\left(=g\left(x_{\sigma}\right)\right)$ for all $\sigma$, it follows by continuity that $\bar{h}\left(t^{\prime \prime}\right)=\bar{h}^{\prime}\left(t^{\prime \prime}\right)$. Hence $g$ extends to all of $W$ in the required way.

(ii) If now $V$ is not the Stone-Čech compactification of $U$, there exists a complexvalued function $g$, defined, bounded, and continuous on $U$, which has no extension to a continuous function on $V$. Suppose that for each point $t$ of $V \backslash U$ there exists some neighbourhood $W_{t}$ of $t$ such that $g$ extends to a bounded continuous function on $W_{t}$. Arguing as in the last paragraph of (i) above, we can piece together these extensions to get a continuous extension of $g$ on all of $V$, which is supposed impossible. This contradiction shows that there exists $t_{0}$ in $V \backslash U$ such that $g$ does not extend to a continuous function on any neighbourhood of $t_{0}$.

THEOREM 2. Suppose that $\partial X \backslash Z$ is empty. Then a necessary and sufficient condition that every derivation on $A$ can be implemented by an operator field continuous with respect to $A$ on $T$ is that $\bar{X}$ be the Stone-Čech compactification of $X$.

Proof. (i) Suppose that $\bar{X}$ is the Stone-Čech compactification of $X$. Let $D$ be any derivation on $A$. We have already shown that $D$ can be implemented by a bounded operator field $b$ on $T$ which is continuous with respect to $A$ on $X \cup Y$. Suppose $t_{0} \in Z$, with $t_{0} \in O^{\mu}$ say. Then for all $t \in V^{\mu \gamma} \subseteq O^{\mu} \cap X$ we have

$$
\begin{aligned}
\left(D e_{11}^{\mu}\right)(t) & =\sum_{i, j=1,2} \beta_{i j}^{\mu \gamma}(t) e_{i j}^{\mu \gamma}(t) e_{11}^{\mu}(t)-\sum_{i, j=1,2} e_{11}^{\mu}(t) \beta_{i j}^{\mu \gamma}(t) e_{i j}^{\mu \gamma}(t) \\
& =\beta_{21}^{\mu \gamma}(t) e_{21}^{\mu \gamma}(t)-\beta_{12}^{\mu \gamma}(t) e_{12}^{\mu \gamma}(t) .
\end{aligned}
$$

Applying the continuity conditions given in $\$ 2$ for points of $Z$ to the element $D e_{11}^{\mu}$ of $A$ and the point $t_{0}$, we deduce that condition (iia) is already satisfied by $b$. Since the centre of $A_{t_{0}}$ is all of $A_{t_{0}}\left(\cong M_{1} \oplus M_{1}\right)$, condition (ia) will be satisfied if we can redefine $\beta_{11}^{\mu}\left(t_{0}\right)$, 
$\beta_{22}^{\mu}\left(t_{0}\right)$ to make $\beta_{11}^{\mu}, \beta_{22}^{\mu}$ continuous at $t_{0}$. If this can be done for all $t_{0}$ in $O^{\mu} \cap Z$, and in a way consistent on 'overlaps' of the form $O^{\mu} \cap O^{\nu} \cap Z$, then $b$ will be modified into an operator field continuous with respect to $A$ on all of $T$ and still implementing $D$. Now $b$ is bounded, so $\beta_{11}^{\mu}$ and $\beta_{22}^{\mu}$ are bounded, and already continuous on $O^{\mu} \cap X$; so, by Lemma 2(i), $\beta_{11}^{\mu} \mid\left(O^{\mu} \cap X\right)$ (and likewise $\beta_{22}^{\mu} \mid\left(O^{\mu} \cap X\right)$, which is just $-\beta_{11}^{\mu} \mid\left(O^{\mu} \cap X\right)$ by the zero trace construction already carried out in $X$ ) extends to a bounded continuous function defined on all of $O^{\mu}$. We use these extensions to redefine $\beta_{11}^{\mu}$ and $\beta_{22}^{\mu}$ continuously on $O^{\mu} \cap Z$; and all that remains is to check consistency on overlaps.

Suppose then that $t_{0} \in O^{\mu} \cap O^{\nu} \cap Z$. As explained in $\S 2$, either $e_{11}^{\mu}\left(t_{0}\right)=e_{11}^{\nu}\left(t_{0}\right)$ and $e_{22}^{\mu}\left(t_{0}\right)=e_{22}^{\nu}\left(t_{0}\right)$, in which case we must prove that $\beta_{11}^{\mu}\left(t_{0}\right)=\beta_{11}^{\nu}\left(t_{0}\right)$ and $\beta_{22}^{\mu}\left(t_{0}\right)=\beta_{22}^{\nu}\left(t_{0}\right)$; or $e_{11}^{\mu}\left(t_{0}\right)=e_{22}^{\nu}\left(t_{0}\right)$ and $e_{22}^{\mu}\left(t_{0}\right)=e_{11}^{\nu}\left(t_{0}\right)$, in which case we must prove that $\beta_{11}^{\mu}\left(t_{0}\right)=\beta_{22}^{\nu}\left(t_{0}\right)$ and $\beta_{22}^{\mu}\left(t_{0}\right)=\beta_{11}^{\nu}\left(t_{0}\right)$. We will deal with the former possibility: a similar proof covers the latter. We need then only show that $\beta_{11}^{\mu}\left(t_{0}\right)=\beta_{11}^{\nu}\left(t_{0}\right)$, since $\beta_{22}^{\mu}\left(t_{0}\right)=-\beta_{11}^{\mu}\left(t_{0}\right)$ and $\beta_{22}^{\nu}\left(t_{0}\right)=$ $-\beta_{11}^{\nu}\left(t_{0}\right)$ by construction.

Let $\left(x_{\sigma}\right)$ be a net in $O^{\mu} \cap O^{\nu} \cap X$ converging to $t_{0}$. Then $x_{\sigma} \in V^{\mu \nu} \cap V^{\nu \delta}$ for some $\gamma, \delta$ depending on $\sigma$, and we may write

$$
b\left(x_{\sigma}\right)=\sum_{i, j=1,2} \beta_{i j}^{\mu \gamma}\left(x_{\sigma}\right) e_{i j}^{\mu \gamma}\left(x_{\sigma}\right)=\sum_{i, j=1,2} \beta_{i j}^{\nu \delta}\left(x_{\sigma}\right) e_{i j}^{\nu \delta}\left(x_{\sigma}\right)
$$

We may also write

$$
e_{11}^{\nu}\left(x_{\sigma}\right)=\sum_{i, i=1,2} \varepsilon_{i j}^{\mu \gamma}\left(x_{\sigma}\right) e_{i j}^{\mu \gamma}\left(x_{\sigma}\right)
$$

Since $e_{11}^{\mu}\left(t_{0}\right)=e_{11}^{\nu}\left(t_{0}\right)$, it follows from continuity conditions (ia) and (iia) of $\S 2$, applied to $e_{11}^{\nu}$, that

$$
\varepsilon_{11}^{\mu}\left(x_{\sigma}\right) \stackrel{\sigma}{\rightarrow} 1 \text { and all other }\left|\varepsilon_{i j}^{\mu}\left(x_{\sigma}\right)\right| \stackrel{\sigma}{\rightarrow} 0
$$

Now $e_{11}^{\nu}\left(x_{\sigma}\right) b\left(x_{\sigma}\right) e_{11}^{\nu}\left(x_{\sigma}\right)$ can be written as

$$
\left(\sum_{i, j=1,2} \varepsilon_{i j}^{\mu \gamma}\left(x_{\sigma}\right) e_{i j}^{\mu \gamma}\left(x_{\sigma}\right)\right)\left(\sum_{i, j=1,2} \beta_{i j}^{\mu \gamma}\left(x_{\sigma}\right) e_{i j}^{\mu \nu}\left(x_{\sigma}\right)\right)\left(\sum_{i, j=1,2} \varepsilon_{i j}^{\mu \gamma}\left(x_{\sigma}\right) e_{i j}^{\mu \gamma}\left(x_{\sigma}\right)\right)
$$

so that, using (1), we find that

$$
\left\|e_{11}^{\nu}\left(x_{\sigma}\right) b\left(x_{\sigma}\right) e_{11}^{\nu}\left(x_{\sigma}\right)-\beta_{11}^{\mu}\left(x_{\sigma}\right) e_{11}^{\mu}\left(x_{\sigma}\right)\right\| \stackrel{\sigma}{\rightarrow} 0
$$

But also

$$
e_{11}^{\nu}\left(x_{\sigma}\right) b\left(x_{\sigma}\right) e_{11}^{\nu}\left(x_{\sigma}\right)=\beta_{11}^{\nu}\left(x_{\sigma}\right) e_{11}^{\nu}\left(x_{\sigma}\right)=\beta_{11}^{\nu}\left(x_{\sigma}\right)\left(\sum_{i, j=1,2} \varepsilon_{i j}^{\mu \gamma}\left(x_{\sigma}\right) e_{i j}^{\mu \gamma}\left(x_{\sigma}\right)\right),
$$

so that, by (1),

$$
\left\|e_{11}^{\nu}\left(x_{\sigma}\right) b\left(x_{\sigma}\right) e_{11}^{\nu}\left(x_{\sigma}\right)-\beta_{11}^{\nu}\left(x_{\sigma}\right) e_{11}^{\mu}\left(x_{\sigma}\right)\right\| \stackrel{\sigma}{\rightarrow} 0
$$


From (2) and (3),

$$
\left\|\beta_{11}^{\mu}\left(x_{\sigma}\right) e_{11}^{\mu}\left(x_{\sigma}\right)-\beta_{11}^{\nu}\left(x_{\sigma}\right) e_{11}^{\mu}\left(x_{\sigma}\right)\right\|=\left|\beta_{11}^{\mu}\left(x_{\sigma}\right)-\beta_{11}^{\nu}\left(x_{\sigma}\right)\right| \stackrel{\sigma}{\rightarrow} 0 .
$$

But by construction $\beta_{11}^{\mu}\left(x_{\sigma}\right) \stackrel{\sigma}{\rightarrow} \beta_{11}^{\mu}\left(t_{0}\right)$ and $\beta_{11}^{\nu}\left(x_{\sigma}\right) \stackrel{\sigma}{\rightarrow} \beta_{11}^{\nu}\left(t_{0}\right)$, so that $\beta_{11}^{\mu}\left(t_{0}\right)=\beta_{11}^{\nu}\left(t_{0}\right)$ as required.

(ii) Suppose that $\bar{X}$ is not the Stone-Čech compactification of $X$. By Lemma 2 (ii) there exists a bounded continuous complex-valued function $g$ defined on $X$ and a point $t_{0}$ in $Z$ such that $g$ cannot be extended to a bounded continuous function on any neighbourhood of $t_{0}$.

Suppose $t_{0} \in O^{\mu}$. Since $\bar{X}$ is compact Husdorff, arguments like those used at the beginning of the proof of Lemma 2 (i) show the existence of an open neighbourhood $G$ of $t_{0}$ in $\bar{X}$ with $\bar{G} \subseteq O^{\mu}$, and of a function $h$, defined, bounded, and continuous on $X$, which agrees with $g$ on $\bar{G} \cap X$ and vanishes outside $O^{\mu}$. Since $g$ does not extend to a bounded continuous function on $G \cap Z$, neither does $h$.

Define an operator field $b$ on $T$ by putting

$$
b(t)= \begin{cases}h(t) e_{11}^{\mu}(t) & \left(t \in O^{\mu} \cap X\right) \\ 0 & \left(t \in T \backslash\left(O^{\mu} \cap X\right)\right)\end{cases}
$$

(This operator field is not continuous with respect to $A$ at points of $G \cap Z$.) We show that $b a-a b$ belongs to $A$ whenever $a$ does. Let $a \in A$, with

$$
a(t)=\sum_{i, j=1,2} \alpha_{i j}^{\mu \gamma}(t) e_{i j}^{\mu \gamma}(t) \quad\left(t \in V^{\mu \gamma}\right)
$$

Then

$$
b(t) a(t)-a(t) b(t)= \begin{cases}\alpha_{12}^{\mu \gamma}(t) h(t) e_{12}^{\mu \gamma}(t)-\alpha_{21}^{\mu \gamma}(t) h(t) e_{21}^{\mu \gamma}(t) & \left(t \in V^{\mu \gamma} \subseteq O^{\mu} \cap X\right) \\ 0 & \left(t \in T \backslash\left(O^{\mu} \cap X\right)\right) .\end{cases}
$$

Clearly the operator field $b a-a b$ satisfies the separation conditions of Lemma 1 , since $b(t) a(t)-a(t) b(t)=0$ for all $t \in Y \cup Z$; and it is not hard to check the relevant continuity conditions of $\S 2$ for $b a-a b$ : the only problems might occur on the boundary of $O^{\mu}$, but these disappear when we observe that $\left|\alpha_{12}^{\mu \gamma}(t)\right|$ and $\left|\alpha_{21}^{\mu \gamma}(t)\right|$ become small for $t$ near $O^{\mu} \cap Z$ (by condition (iia) applied to $a$ ) and that $h(t)$ becomes small for $t$ near the other points of the boundary of $O^{\mu}$.

Thus $b$ implements a derivation $D$ on $A$. Suppose that $D$ can be implemented by an operator field $c$ which is continuous with respect to $A$ on $T$, and therefore in particular on $O^{\mu}$. The continuity conditions of $\$ 2$, together with the fact that, for each $t$ in $T$, $b(t)-c(t)$ belongs to the centre of $A_{t}$, shows that there must exist a (bounded continuous) complex-valued function $\theta$ defined on $O^{\mu} \cap X$ such that the functions $h-\theta$ and $-\theta$ on $O^{\mu} \cap X$ both extend to bounded continuous functions on $O^{\mu} \cap Z$, and in particular on $G \cap Z$. But then by subtraction the same would be true of $h$, a contradiction. This completes the proof of the theorem. 
Corollary. Suppose $\partial X \backslash Z$ is empty. Then if $\bar{X}$ is not the Stone-Čch compactification of $X$, there exists an outer derivation on $A$.

We show finally how a derivation $D$ can arise which can be implemented by an operator field continuous with respect to $A$ on $T$, but not by one which also satisfies the separation conditions of Lemma 1 (so that $D$ is outer).

Lemma 3. Let $D$ be a derivation on $A$, implemented by each of two operator fields $b, c$ on $T$. If both $b$ and $c$ are continuous with respect to $A$ on $T$, then $b(t)-c(t)$ is a scalar multiple of the identity $I_{t}$ of $A_{t}$ for all $t$ in $T$.

Proof. Put $d=b-c$, so that $d$ is continuous with respect to $A$ on $T$. We already know that $d(t)=\delta(t) I_{t}$ (for some complex number $\delta(t)$ ) for $t \in T \backslash Z$.

Let $t_{0} \in Z$, with $t_{0} \in O^{\mu}$. The continuity condition (ia) of $\$ 2$, applied to $d$, shows that the coefficient functions $\delta_{11}^{\mu}$ and $\delta_{22}^{\mu}$, and hence also $\delta_{11}^{\mu}-\delta_{22}^{\mu}$, are continuous at $t_{0}$. For $t \in O^{\mu} \cap X, \delta_{11}^{\mu}(t)-\delta_{22}^{\mu}(t)=\delta(t)-\delta(t)=0$; and so, by continuity, $\delta_{11}^{\mu}\left(t_{0}\right)-\delta_{22}^{\mu}\left(t_{0}\right)=0$. Thus $d\left(t_{0}\right)$ is a scalar multiple of $I_{t_{0}}$, as required.

Recall now that we write the elements of $\partial X$ in the form $\left[y_{1}, y_{2}\right]$ as unordered pairs of points of $Y$.

DEFINITION. (1) If $y_{1}, y_{2}$ are (not necessarily distinct) elements of $Y$, we say that $y_{1}$ links with $y_{2}$ if $\left[y_{1}, y_{2}\right] \in \partial X$.

(2) For any $n \geq 3$, a finite sequence $\left\{y_{1}, y_{2}, \ldots, y_{n}\right\}$ of points of $Y$ is called a circle in $Y$ if $y_{j}$ links with $y_{j+1}(1 \leq j \leq n-1)$ and $y_{n}$ links with $y_{1}$; if in addition $y_{1}, \ldots, y_{n}$ are all distinct, the circle is called proper.

THEOREM 3. Suppose there exists a proper circle in $Y$. Then there exists a derivation $D$ on $A$ which can be implemented by an operator field continuous with respect to $A$ on $T$, but not by one which also satisfies the separation conditions of Lemma 1 (so that D is outer).

Proof. Let $\left\{y_{1}, y_{2}, \ldots, y_{n}\right\}(n \geq 3)$ be a proper circle in $Y$, and put $z_{1}=\left[y_{1}, y_{2}\right]$, $z_{2}=\left[y_{2}, y_{3}\right], \ldots, z_{n}=\left[y_{n}, y_{1}\right]$. Since $y_{1}, \ldots, y_{n}$ are all distinct, each $z_{j}$ belongs to $Z$ and hence to some $O^{\mu_{i}} ; z_{1}, \ldots, z_{n}$ are themselves distinct, and so we may assume that the $O^{\mu_{i}}$ are mutually disjoint by shrinking them if necessary. We may also suppose that the corresponding matrix units are chosen so that $e_{11}^{\mu_{1}}\left(y_{j}\right)=e_{22}^{\mu_{1}}\left(y_{i+1}\right)=1, e_{11}^{\mu_{1}}\left(y_{j+1}\right)=e_{22}^{\mu_{i}}\left(y_{j}\right)=0$ $(1 \leq j \leq n-1)$ and $e_{11}^{\mu_{n}}\left(y_{n}\right)=e_{22}^{\mu_{n}}\left(y_{1}\right)=1, e_{11}^{\mu_{n}}\left(y_{1}\right)=e_{22}^{\mu_{n}}\left(y_{n}\right)=0$.

There exist continuous complex-valued functions $f_{i}(j=1, \ldots, n)$ on $\bar{X}$ such that $f_{i}\left(z_{j}\right)=1$ and $f_{j}(t)=0$ for $t \in \bar{X} \backslash O^{\mu_{i}}$. Define an operator field $b$ on $T$ by putting

$$
b(t)= \begin{cases}f_{j}(t) e_{11}^{\mu_{j}}(t) & \left(t \in O^{\mu_{i}}, j=1, \ldots, n\right) \\ 0 & \left(t \in T \backslash \bigcup_{i=1}^{n} O^{\mu_{i}}\right) .\end{cases}
$$

Since the $O^{\mu_{i}}$ are mutually disjoint and each $f_{j}$ is continuous and vanishes outside $O^{\mu_{i}}, b$ is well-defined and continuous with respect to $A$ on $T$. For each $a \in A, b a-a b$ belongs to $A$, 
for it is continuous with respect to $A$ on $T$ (since $b$ is) and satisfies the separation conditions of Lemma 1 (because $b(t) a(t)-a(t) b(t)=0$ for all $t$ in $\partial X \cup Y$ ).

Thus $b$ implements a derivation $D$ on $A$. Suppose that $D$ can be implemented by an operator field $c$ which is continuous with respect to $A$ on $T$ and also satisfies the separation conditions of Lemma 1. For each $t$ in $T, c(t)=b(t)-\delta(t) I_{t}$ for some complex number $\delta(t)$, by Lemma 3. Denoting by $\gamma\left(\bar{s}_{1}, s_{2}\right), \gamma\left(s_{1}, \bar{s}_{2}\right)$ the coefficients of $c$ at $\left[s_{1}, s_{2}\right]$ corresponding to $s_{1}, s_{2}$ respectively (see $\$ 2$ ), the first of the four separation conditions of Lemma 1, applied to $c$ for the points $z_{1}, \ldots, z_{n}$, gives

$$
\begin{aligned}
& \gamma\left(\bar{y}_{1}, y_{2}\right)=f_{1}\left(z_{1}\right)-\delta\left(z_{1}\right)=-\delta\left(z_{n}\right)=\gamma\left(y_{n}, \bar{y}_{1}\right) \\
& \gamma\left(\bar{y}_{2}, y_{3}\right)=f_{2}\left(z_{2}\right)-\delta\left(z_{2}\right)=-\delta\left(z_{1}\right)=\gamma\left(y_{1}, \bar{y}_{2}\right) \\
& \cdot \\
& \cdot \\
& \cdot \\
& \gamma\left(\bar{y}_{n}, y_{1}\right)=f_{n}\left(z_{n}\right)-\delta\left(z_{n}\right)=-\delta\left(z_{n-1}\right)=\gamma\left(y_{n-1}, \bar{y}_{n}\right) .
\end{aligned}
$$

But $f_{j}\left(z_{i}\right)=1(j=1, \ldots, n)$, so by addition we get $n=0$, a contradiction. This completes the proof of the theorem.

I can prove that if there are no proper circles in $Y$ and certain other conditions are also satisfied, then any derivation $D$ on $A$ which can be implemented by an operator field continuous with respect to $A$ on $T$ can be implemented by one which also satisfies the separation conditions of Lemma 1 (so that $D$ is inner). The extra conditions are not very satisfactory (examples show them not to be necessary conditions). It is hoped to pursue these matters further on another occasion; there is also the possibility of using Vasil'ev's structure theory to investigate derivations on $\mathrm{C}^{*}$-algebras with irreducible *representations of bounded finite dimension, but with a bound higher than 2 .

\section{REFERENCES}

1. J. Dixmier, Les algèbres d'opérateurs dans l'espace Hilbertien (Algèbres de von Neumann), 2nd edition (Gauthier-Villars, 1969).

2. J. Dixmier, Les $C^{*}$-algèbres et leurs représentations, 2nd edition (Gauthier-Villars, 1969).

3. G. A. Elliott, Some $C^{*}$-algebras with outer derivations, Rocky Mountain J. Math. 3 (1973), 501-506.

4. G. A. Elliott, Some C*-algebras with outer derivations, III, Ann. of Math. 106 (1977), 121-143.

5. J. M. G. Fell, The structure of algebras of operator fields, Acta Math. 106 (1961), 233-280.

6. J. P. Sproston, Derivations and automorphisms of homogeneous $\mathrm{C}^{*}$-algebras, Proc. London Math. Soc. (3) 32 (1976), 521-536.

7. N. B. Vasil'ev, $\mathrm{C}^{*}$-algebras with finite-dimensional irreducible representations, Russian Math. Surveys 21 (1966), 137-155. 
8. C. A. Akemann and G. K. Pedersen, Central sequences and inner derivations of separable C*-algebras, Amer. J. Math. 101 (1979), 1047-1061.

Department of Pure Mathematics,

UNIVERSTTY OF HULL,

22/24, Newland Park,

Cottingham Road,

Hull,

HU5 2DW. 